\title{
RAVL1 Activates Brassinosteroids and Ethylene Signaling to Modulate Response to Sheath Blight Disease in Rice
}

\author{
De Peng Yuan, Chong Zhang, Zi Yuan Wang, Xiao Feng Zhu, and Yuan Hu Xuan ${ }^{\dagger}$
}

All authors: College of Plant Protection, Shenyang Agricultural University, Shenyang, Liaoning, 110866 China.

Accepted for publication 16 April 2018.

\begin{abstract}
Rhizoctonia solani causes sheath blight disease in rice; however, the defense mechanism of rice plants against $R$. solani remains elusive. To analyze the roles of brassinosteroid (BR) and ethylene signaling on rice defense to $R$. solani, wild-type (WT) rice and several mutants and overexpressing $(\mathrm{OX})$ lines were inoculated with $R$. solani. Mutants $d 61-1$ and $d 2$ were less susceptible to sheath blight disease, bril-D was more susceptible, and ravl1 and d61-1/EIL1 Ri5 were similarly susceptible compared with WT. The double mutant ravl1/d61-1 was phenotypically similar to the ravll mutant. Transcriptome analysis, chromatin immunoprecipitation assay, electrophoretic mobility shift assay, and transient

assays indicted that RAVL1 might directly activate Ethylene insensitive 3like 1 (EIL1), a master regulator of ethylene signaling. Mutants ers 1 and d61-1/RAVL1 OX were resistant to sheath blight disease, whereas EIL1 RNAi mutants and RAVL1 OX were more susceptible than WT. BRI1 and D2 expression in EIL1 Ri5/RAVL1 OX and EIL1 expression in $d 61$ 1/RAVL1 OX indicated that RAVL1 activates BRI1/D2 and EIL1, respectively, independent of BR and ethylene signaling. Our analyses provide information on how BR and ethylene signaling regulate sheath blight disease and on the regulatory function of RAVL1 in rice sheath blight disease.
\end{abstract}

Sheath blight is one of the three major diseases in rice (Oryza sativa L.) and is caused by the fungal pathogen Rhizoctonia solani (Savary et al. 1995). Sheath blight threatens rice throughout its growth cycle, from seedling to heading, and causes lesions on leaves, sheaths, and panicles. At the late stage of infection, the whole plant withers and lodges (Savary et al. 1995). Sheath blight can reduce rice yield by 8 to $50 \%$, depending on disease severity, crop stage at which the infection occurs, and environmental conditions (Savary et al. 2000). Currently, chemicals are extensively used as the main strategy for disease control (Savary et al. 2000), albeit increasing health risks, production cost, and environmental pollution. Thus, plant resistance based on host-pathogen interaction is considered a hot spot of research. Previous studies have shown that overexpression of chitinase, $\beta$-1,3-glucanase, and polygalacturonase inhibiting protein (OsPGIP1) enhances rice resistance against $R$. solani (Mao et al. 2014; Shah et al. 2009; Wang et al. 2015).

Brassinosteroids (BRs) are a major category of phytohormones. In Arabidopsis thaliana, their signaling starts with BRASSINOSTEROID INSENSITIVE 1 (BRI1) receptor, which leads to the dissociation of BRI1 kinase inhibitor 1 (BKI1) and association of BRI1-ASSOCIATED RECEPTOR KINASE 1 (BAK1). The activation of BRI1-BAK1 ultimately results in the dephosphorylation and activation of BRASSINAZOLE-RESISTANT 1 (BZR1) and BRI1-EMS-SUPPRESSOR 1 (BES1), two transcription factors that regulate target gene expression in response to BRs (Kim and Wang 2010; Li and Chory 1997; Li et al. 2002; Nam and Li 2002;

\section{${ }^{\dagger}$ Corresponding author: Y. H. Xuan; E-mail: xuanyuanhu115@syau.edu.cn}

Funding: This work was supported by grants from the National Natural Science Foundation of China (31571985 and 31330063), and an initiative grant to Y. H. Xuan (880416008) from Shenyang Agricultural University, as well as Key projects of Liaoning Provincial Department of Education (LSNZD201601).

First and second authors contributed equally to this work.

*The $e$-Xtra logo stands for "electronic extra" and indicates that four supplementary figures and two supplementary tables are published online.

(c) 2018 The American Phytopathological Society
Yang et al. 2011). The stability of BES1 and BZR1 is strongly enhanced in bes1-D and bzrl-D gain-of-function mutants, respectively, and rescues the BR-insensitive dwarf phenotype of the bril BR-receptor mutant (Wang et al. 2002; Yin et al. 2002). In rice, a B3 DNA binding domain protein related to ABI3/VP1-Like 1 (RAVL1) has been identified as an upstream regulator in BR homeostasis, as it binds to the E-box in the promoter sequences of BR-receptor and BR biosynthetic genes (Je et al. 2010). A previous study showed that a mutation at the BR receptor BRII enhances disease resistance in Brachypodium distachyon and barley (Hordeum vulgare) against necrotrophic and hemibiotrophic pathogens showing short asymptomatic phase (Goddard et al. 2014). Additionally, BR application causes $86 \%$ reduction in the severity of Fusarium head blight (FHB) in wheat due to Fusarium culmorum, and 33\% reduction in FHB-associated yield losses in barley (Ali et al. 2013), suggesting that the BR signaling mechanism regulating the response to different pathogens is complex.

Ethylene is a gaseous molecule that plays numerous roles in plant growth, development, and response to environmental stresses (Morgan and Drew 1997; van Loon et al. 2006). In A. thaliana, five endoplasmic reticulum (ER)-localized ethylene receptors, namely ETHYLENE RESPONSE 1 (ETR1), ETR2, ETHYLENE RESPONSE SENSOR 1 (ERS1), ERS2, and ETHYLENE INSENSITIVE 4 (EIN4), have been identified as negative regulator of ethylene signaling (Chang et al. 1993; Hua et al. 1995; Sakai et al. 1998). CONSTITUTIVE TRIPLE RESPONSE (CTR) 1 (Raflike CTR1) is also a key negative regulator of ethylene signaling, which functions downstream of the receptors and upstream of EIN2 (Kieber et al. 1993). In the absence of ethylene, CTR1 directly phosphorylates the C-terminal domain of EIN2 to prevent EIN2-signal transferring from the ER to the nucleus (Ju et al. 2012). Furthermore, the signal is transduced from the ER to the nucleus via the EIN2-EIN3/ ETHYLENE INSENSITIVE 3-LIKE 1 (EIL1) pathway (Ju et al. 2012; Qiao et al. 2012; Wen et al. 2012), in which EIL1 activates downstream signals, including ethylene response factors (ERFs) Yang et al. 2015a). Exogenous application of ethylene induces the expression of pathogenesis-related (PR) genes, such as PRI, PR5, and $P R 10$, in rice (Agrawal et al. 2001). Overexpression of an ERF in the roots of Medicago truncatula (MtERF1-1) enhanced its resistance to $R$. solani anastomosis group (AG)-8 (Anderson et al. 2010), whereas 
overexpression of a gene encoding an ethylene synthesis enzyme in rice (OsACS2) enhanced its resistance to multiple diseases, including sheath blight and blast (Helliwell et al. 2013). However, more research is needed to elucidate the role of BR and ethylene signaling in the regulation of sheath blight disease in rice.

Here, we studied BR action against sheath blight disease using brll, $d 2$, and ravll mutants. The latter was the only susceptible mutant, and thus was further analyzed using transcriptomic, biochemical, genetic, and molecular methods. Results showed that $R A V L 1$ directly activates EILI, an ethylene signaling gene that positively regulates the defense mechanism against sheath blight disease. Taken together, our results provide information on the role of BR and ethylene signaling in the regulation of sheath blight disease as well as the linkage between $\mathrm{BR}$ and ethylene signaling in rice.

\section{MATERIALS AND METHODS}

Plant growth and inoculation. Wild-type (WT) rice (Oryza sativa Japonica, cultivars Dongjin and Nipponbare) and ravll, d61-1, d2, bri1-D, ers1, and EIL1 RNAi mutants, as well as RAVL1, d61-1/RAVL1, and EIL1 Ri5/RAVL1 overexpressing (OX) lines (Je et al. 2010; Xuan et al. 2017; Yang et al. 2015b) were used. All plants were grown under greenhouse conditions (temperature, 23 to $30^{\circ} \mathrm{C}$; relative humidity $80 \%$; and $12 \mathrm{~h}$ light: $12 \mathrm{~h}$ dark) at Shenyang Agricultural University, China, and propagated by selfing. Onemonth-old plants were used for inoculation with $R$. solani AG-1 (Prasad and Eizenga 2008). In brief, a 10-cm-long piece was cut from the second youngest leaf of the main tiller and placed on moistened filter paper in a Petri dish (diameter, $36 \mathrm{~cm}$; height, $2.5 \mathrm{~cm}$ ). Each replicate comprised six leaves, and four replicates per line were used, in a completely randomized design. Colonized potato dextrose agar (PDA) blocks (diameter, $7 \mathrm{~mm}$ ) were excised using a circular cutter and placed on the abaxial surface of each leaf piece (Supplementary Fig. S1). Leaves were incubated in a chamber with continuous light at $25^{\circ} \mathrm{C}$ for $72 \mathrm{~h}$. The filter paper was kept moist with sterile water. After $72 \mathrm{~h}$, the length and width of the lesions within each leaf piece were measured using Image J Fiji software and the approximate percentage of leaf covered with lesions was calculated (Eizenga et al. 2002; Prasad and Eizenga 2008; Savary and Mew 1996).

To analyze $R$. solani AG-1 infection-mediated expression of $B R I 1$ and D2, 1-month-old WT and ravll plants were inoculated with $R$. solani AG-1, and their leaves were sampled after $0,24,48$, and $72 \mathrm{~h}$. Relative fungal biomass was calculated using DNA-based quantitative PCR (qPCR). DNA was extracted from $10-\mathrm{cm}$ rice leaf pieces presenting lesions (one piece per leaf; six leaves from each line at each sampling time) using the cetyltrimethyl ammonium bromide protocol. Relative fungal biomass was then measured based on cycle threshold (CT) values of $R$. solani Phosphogluconate dehydrogenase (RsPDG) and rice genomic ubiquitin (OsUBI) genes, according to $2[\mathrm{CT}(O s U B I)-\mathrm{CT}(R s P D G)]$. The primers used for qPCR are listed in Supplementary Table S1.

Generation of crossing mutants and identification of homozygotes in the offspring. The F8 generation of the cross mutants ravll/d61-1 and d61-1/RAVL1 OX were obtained as previously described (Je et al. 2010). The F3 generation of EIL1 Ri5/d61-1 and EIL1 Ri5/RAVL1 OX plants were used, and offspring homozygosity for each genetic combination was isolated by genotyping 30 individual plants from each line, using gene specific primers (EIL1 R or RAVL1 R) and the vector primer 35S F. If all 30 plants carried the plasmid, the lines were considered homozygous for EIL1 Ri5 and RAVL1 OX. Because $d 61-1$ is a point mutation mutant of BRI1, its homozygotes were isolated via the PCR and genotyping methods previously published (Je et al. 2010).

RNA extraction and quantitative real-time (qRT)-PCR analysis. Total RNA was isolated from 1-month-old seedlings with the RNeasy Plant Mini Kit (Qiagen, Hilden, Germany) and TRIzol reagent (Takara, Dalian, China), and then treated with RQ-RNase free DNase (Promega, Madison, WI) to remove genomic DNA contamination. Complementary DNA was then generated using the GoScript Reverse Transcription kit (Promega), according to the manufacturer's instructions. The qRT-PCR was performed using the BIO-RAD CFX96 Real-time PCR system (Bio-Rad, Hercules, CA) with SYBR-Green (Takara). The values were normalized against Ubiquitin that was used as a housekeeping gene.

RNA deep sequencing and gene ontology (GO) analysis. Total RNA was extracted from 1-month-old WT and ravll-1 plants as described above, and used for RNA sequencing at LC Biotech Co. (Hangzhou, China). Three biological replicates from each line were analyzed; thus, data presented here correspond to mean values, followed by the standard error of the mean, of three independent RNA sequencing experiments. Differentially expressed genes (DEGs) associated with biological process GO terms (Ye et al. 2006) were determined as follows: Fisher's exact test was used

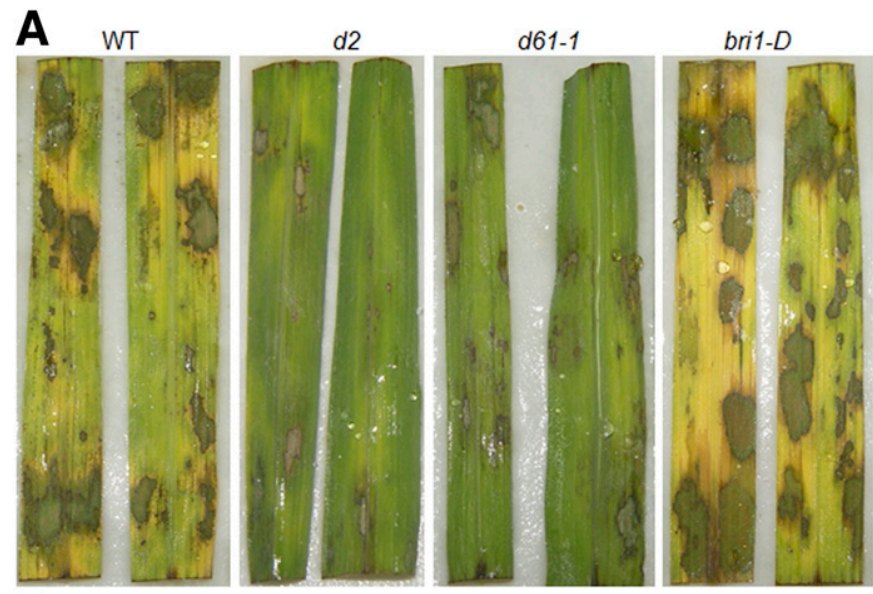

B
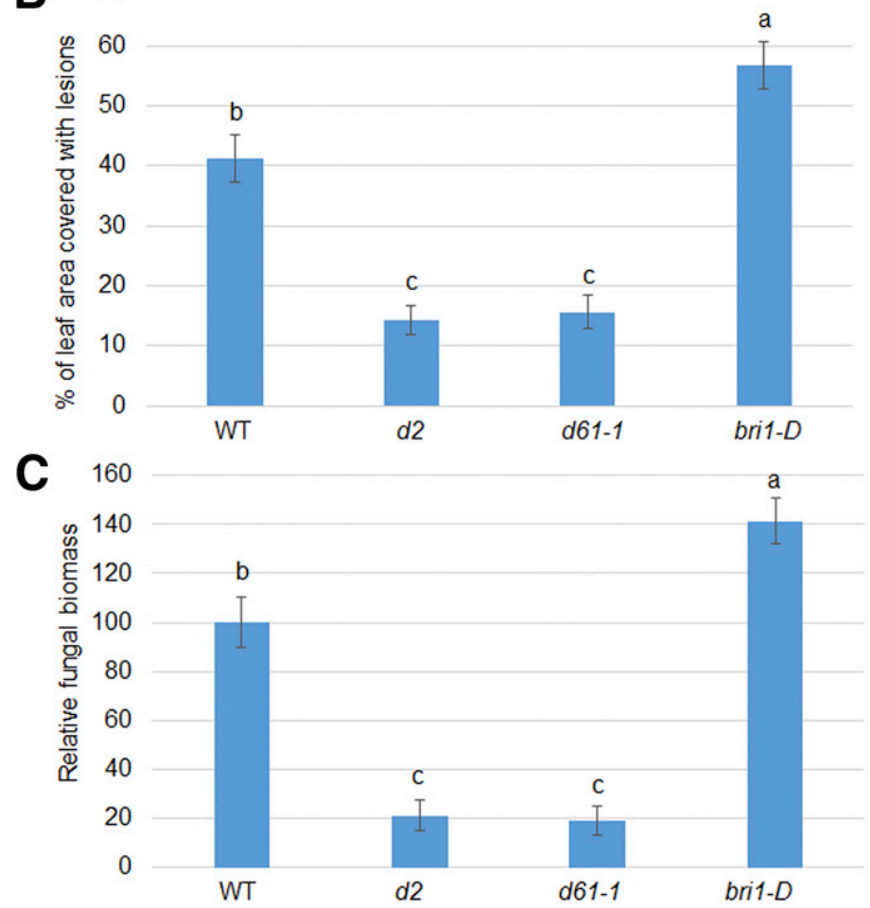

Fig. 1. Evaluation of $d 2, d 61-1$, and bril-D mutants in response to sheath blight disease compared with wild-type (WT) rice (Oryza sativa L.). A, Response of $d 2$, d61-1, and bri1-D to Rhizoctonia solani anastomosis group (AG)-1 compared with WT. B, Percentage of leaf area covered with lesions in $d 2, d 61-1$, and bri1-D compared with WT. Data represent means \pm standard error $(n>10)$. C, Relative fungal biomass obtained by quantitative PCR $\left[2^{[C T(O s U B \mathrm{I})-\mathrm{CT}(R s P G D)]}\right]$ using genomic DNA. The data are means \pm standard error $(n=10)$. Different letters indicate significant differences at $P<0.05$. 
to classify the enrichment of the GO category, and the false discovery rate (FDR) was calculated to correct the $P$ value; the smaller the FDR, the smaller the error in judging $P$ (Dupuy et al. 2007). Enrichment of GO terms among probe sets was found using one-tailed Fisher's exact test based on $2 \times 2$ contingency tables, as it provides a measure of the significance of the enrichment as it increases; the corresponding function is more specific, revealing GOs with a concrete function description in the experiment (Dunnick et al. 2012).

Chromatin immunoprecipitation (ChIP) assay. Rice callus samples $(8 \mathrm{~g})$ collected from transgenic plants expressing $35 \mathrm{~S}$ : RAVL1-green fluorescence protein $(G F P)$ and $35 S: G F P$ were used

A

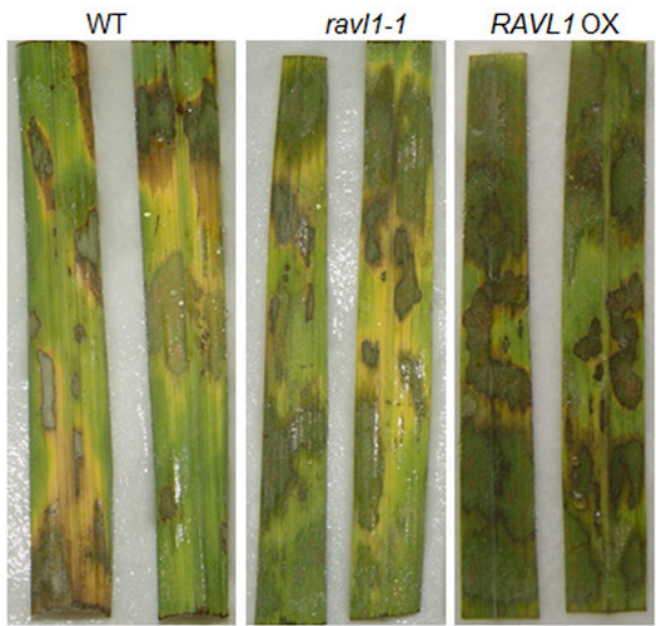

B
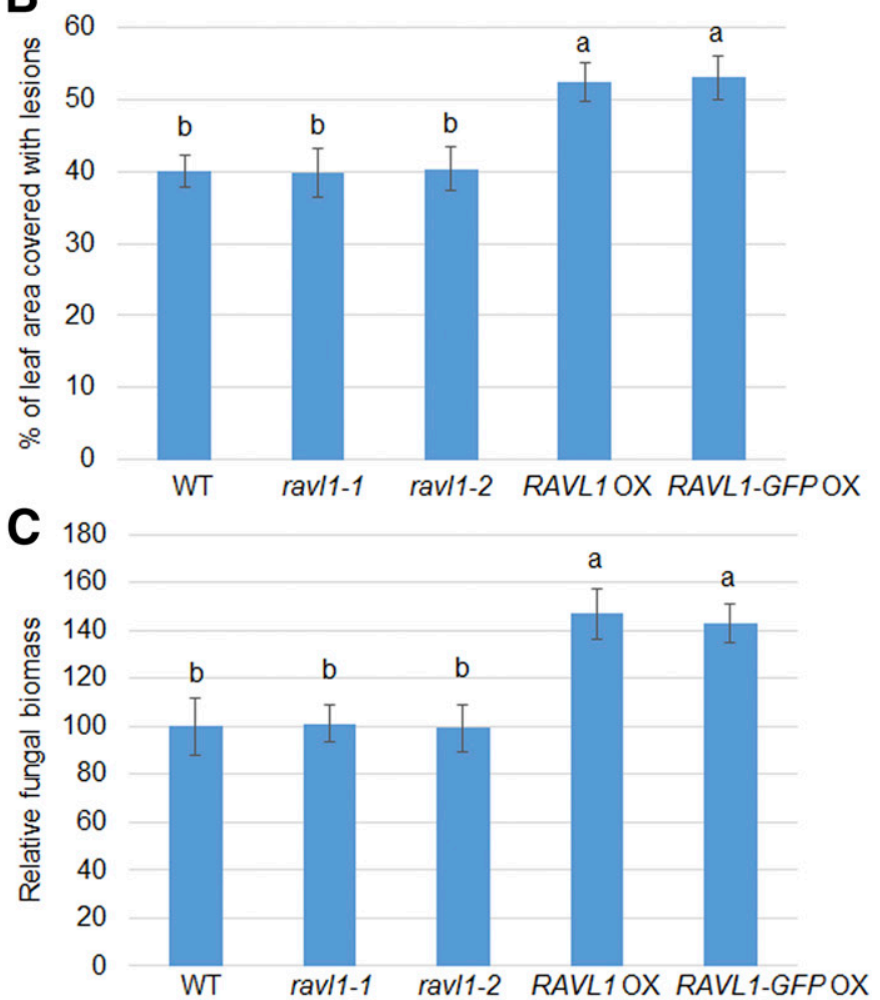

Fig. 2. Evaluation of ravll mutants and RAVL1 overexpression (OX) plants in response to sheath blight disease compared with wild-type (WT) rice (Oryza sativa L.). A, Response of ravl1-1, ravl1-2, RAVL1 OX, and RAVL1-GFP OX to Rhizoctonia solani anastomosis group (AG)-1 compared with WT. B, Percentage of leaf area covered with lesions in ravl1-1, ravl1-2, RAVL1 OX, and RAVL1-GFP OX compared with WT. Data represent means \pm standard error $(n>10)$. C, Relative fungal biomass obtained by quantitative PCR $\left[2^{[\mathrm{CT}(O s U B I)-\mathrm{CT}(R s P G D)]}\right]$ using genomic DNA. The data are means \pm standard error $(n=10)$. Different letters indicate significant differences at $P<0.05$. for ChIP assay. Preabsorption with a preimmune serum was performed before immunoprecipitation with an anti-GFP monoclonal antibody (Clontech, Mountain View, CA). Immunoprecipitates were analyzed by qRT-PCR. Each input DNA level was used as a control to normalize the contents of immune-precipitated DNA in the qRT-PCR assay (Je et al. 2010).

Electrophoretic mobility shift assay (EMSA). To produce RAVL1 recombinant proteins, the open reading frame sequence of $R A V L 1$ was subcloned into the pET28a expression vector, and the resulting $p E T 28 a-R A V L 1$ plasmid was used for the transformation of Escherichia coli strain BL21 DE3. Recombinant proteins were harvested after a 3 -h treatment with $0.4 \mathrm{mM}$ isopropyl
A

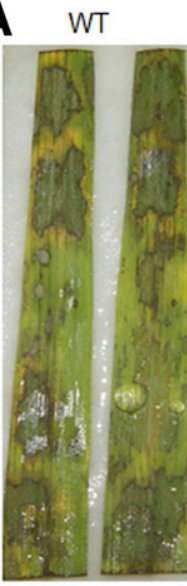

ravl1

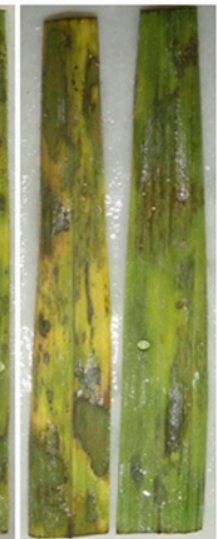

$d 61-1$

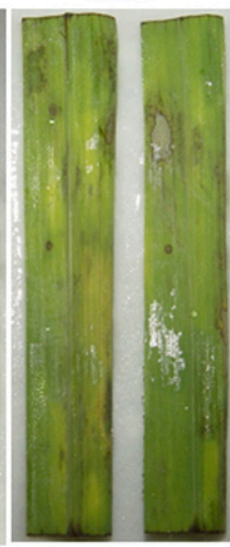

ravl1/d61-1

B 60

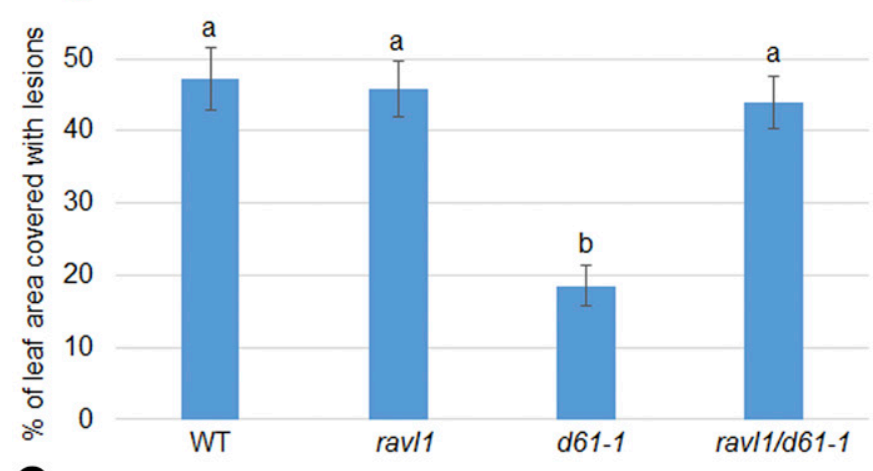

C 120
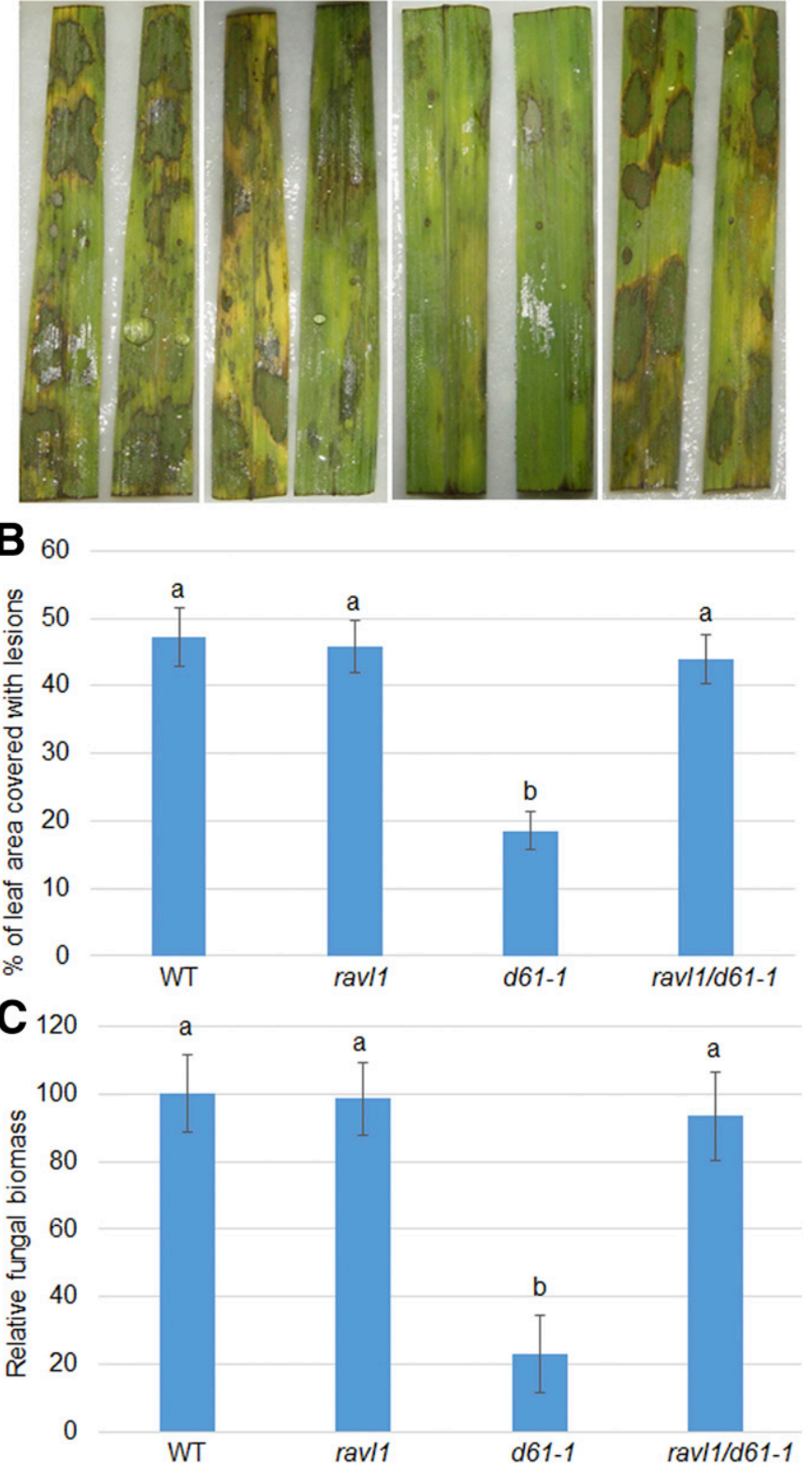

Fig. 3. Evaluation of ravl1/d61-1 double mutant in response to sheath blight disease compared with wild-type (WT) rice (Oryza sativa L.) and parental lines. A, Response of ravl1/d61-1 (F8) to Rhizoctonia solani anastomosis group (AG)-1 compared with WT and parental lines. B, Percentage of leaf area covered with lesions in ravll/d61-1 compared with WT and parental lines. Data represent means \pm standard error $(n>10)$. C, Relative fungal biomass obtained by quantitative PCR [2[CT(OsUBI)-CT(RsPGD)]$]$ using genomic DNA. The data are means \pm standard error $(n=10)$. Different letters indicate significant differences at $P<0.05$. 
$\beta$-D-1-thiogalactopyranoside at $27^{\circ} \mathrm{C}$. For EMSA, a standard binding reaction was performed in a total volume of $20 \mu \mathrm{l}$ by incubating $1 \mu \mathrm{g}$ of purified protein, $40,000 \mathrm{cpm} 32 \mathrm{P}$-labeled DNA probe, and $1 \mu \mathrm{g}$ of poly dI-dC, which blocks any nonspecific binding between protein and probe DNA, in a reaction buffer $(25 \mathrm{mM}$ HEPES-KOH, $\mathrm{pH} 7.5 ; 100 \mathrm{mM} \mathrm{KCl} ; 0.1 \mathrm{mM}$ ethylenediaminetetraacetic acid [EDTA], $10 \%$ [vol/vol] glycerol, and $1 \mathrm{mM}$ dithiothreitol) at $25^{\circ} \mathrm{C}$ for $30 \mathrm{~min}$. The products were resolved in $8 \%$ polyacrylamide gel electrophoresis in $0.5 \times$ Tris/Borate/EDTA buffer (Je et al. 2010). For probe labeling, 20-nucleotide DNA fragments were synthesized and end-labeled with $[\gamma-32 \mathrm{P}]$-labeled ATP using T4 polynucleotide kinase (NEB, Ipswich, MA). For the competition assay, the recombinant RAVL1 protein was incubated with 50-fold excess unlabeled $\mathrm{P} 2$ and $\mathrm{mP} 2$ probes for $30 \mathrm{~min}$ at $25^{\circ} \mathrm{C}$, before adding labeled probes.

Transient expression assay. Constructs, harboring an effector (35S:RAVL1), reporter ( $p E I L 1)$, mutated promoter ( $m p E I L 1)$, and internal control (35S:LUC), were used for the cotransformation of A. thaliana protoplasts (Yamaguchi et al. 2010). To generate $p 35 S$ : $R A V L 1$, the GAL4BD region of the p35S:GAL4BD vector was replaced by $R A V L 1$ cDNA (Xuan et al. 2017). To obtain the reporter vectors, a 1.5-kb EIL1 promoter sequence was constructed. In the mutated promoter, E-box element sequences (CANNTG) were substituted by the TTTTTT sequence. Native and mutated promoters of EIL1 were cloned into the p35S:TATA:GUS vector by replacing the 35S:TATA region with EIL1 promoters. Plasmids $35 S$ : RAVL1 and pEIL1-GUS or 35S:RAVL1 and mpEIL1-GUS $(2 \mu \mathrm{g})$ were transformed into $A$. thaliana protoplasts via polyethylene glycol (PEG)-mediated transformation method. After 12 and $24 \mathrm{~h}$ of transformation, protoplast cells were harvested and proteins were extracted. $\beta$-Glucuronidase (GUS) activity was tested using the GUS assay kit (Promega), and its expression was normalized against luciferase (LUC) expression (Xuan et al. 2013). Luciferase was assayed using the Luciferase assay kit (Promega). Both PEG-mediated transformation and activity assays were performed as previously described (Yoo et al. 2007).

Statistical analyses. Statistical analyses were performed using Prism 5 (GraphPad, San Diego, CA). All data are expressed as means \pm standard error (SE). One-way analysis of variance (ANOVA) was performed, followed by Bonferroni's multiple comparison tests. Differences among samples were considered significant at $P<0.05$.

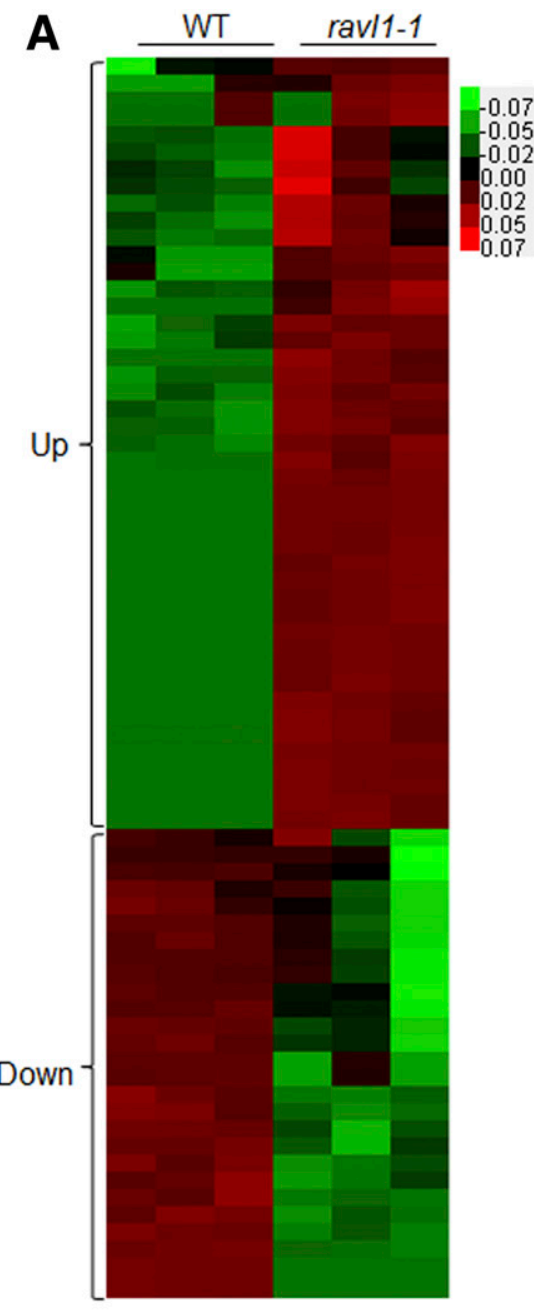

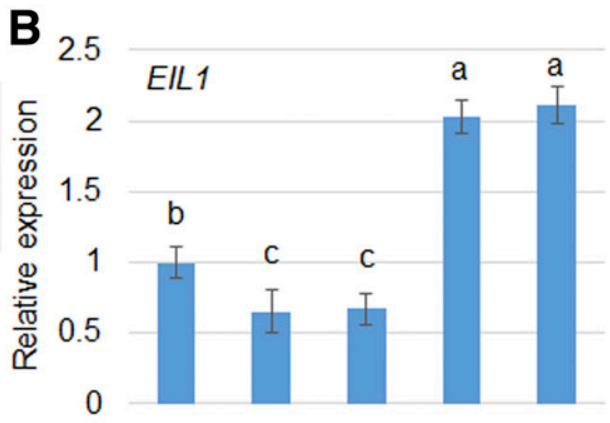
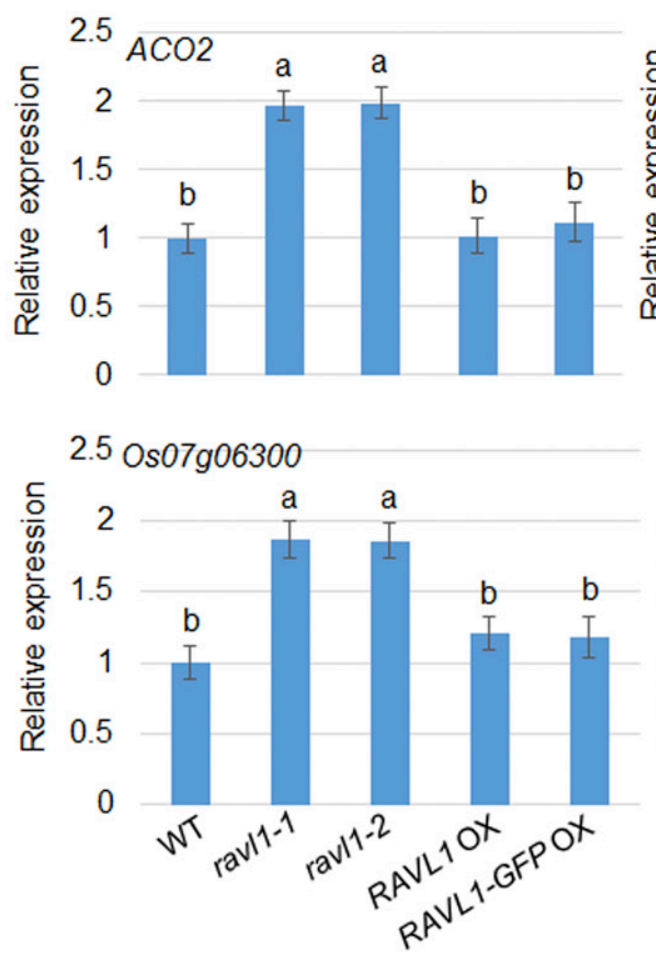
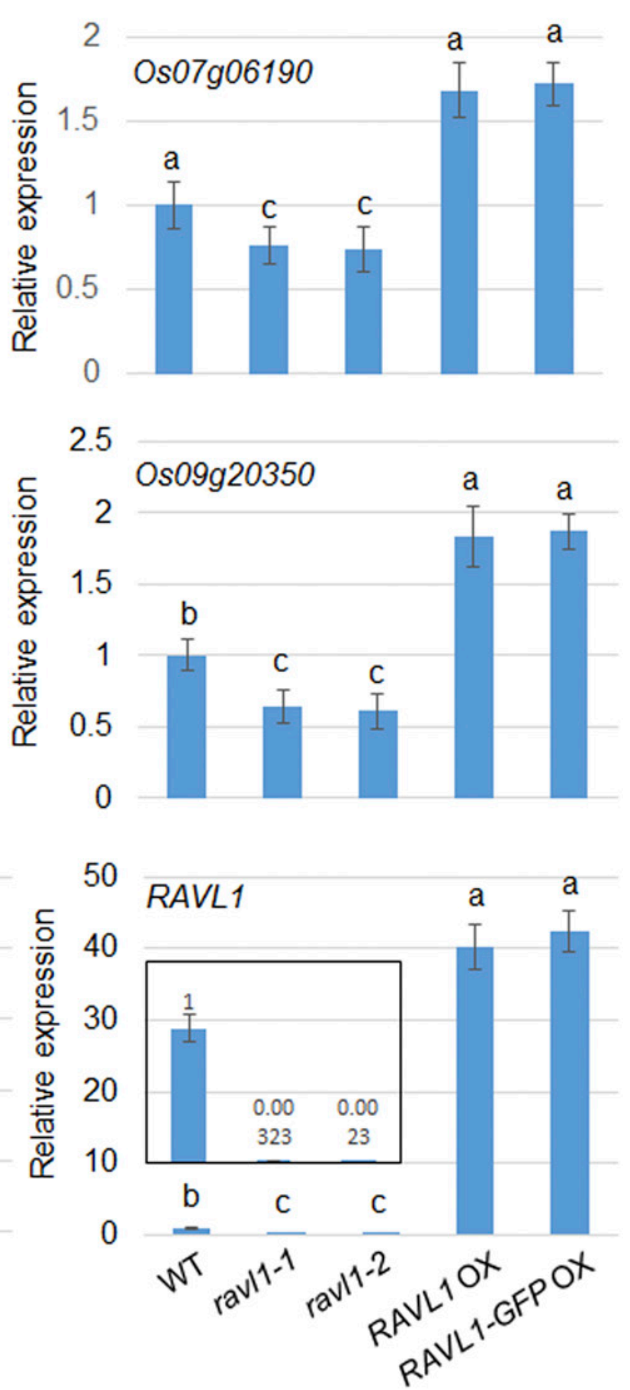

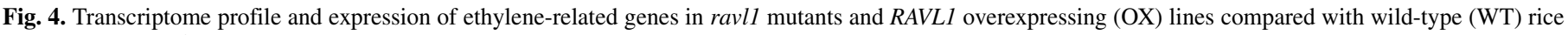

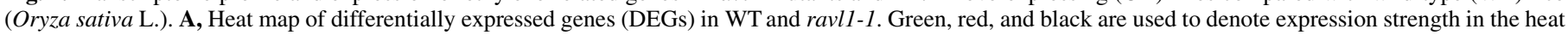

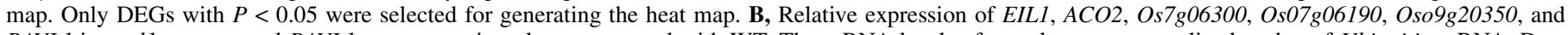

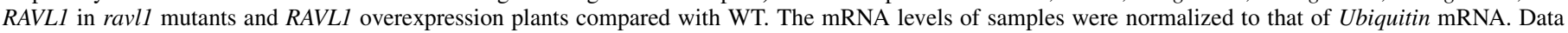

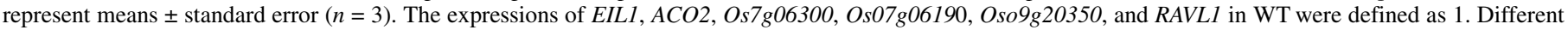
letters indicate significant differences at $P<0.05$. 


\section{RESULTS}

BR signaling negatively regulates rice resistance to sheath blight disease. To investigate the $\mathrm{BR}$ function in rice resistance against $R$. solani AG-1, WT and bril mutant d61-1 (Yamamuro et al. 2000), BR biosynthesis deficient mutant $d 2$ (Hong et al. 2003), and BRII OX mutant bril-D (Jeong et al. 2002) were inoculated with the fungus, and the percentage of leaf area covered with lesions was estimated. Results showed that the $d 61-1$ and $d 2$ were more resistant to $R$. solani AG-1 than WT plants, whereas bril-D showed more severe symptoms than WT plants (Fig. 1A). The percentage of leaf area covered with lesions was $40 \%$ in WT, $15 \%$ in $d 61-1,13 \%$ in $d 2$, and $56 \%$ in bril-D (Fig. 1B). Relative fungal biomass $72 \mathrm{~h}$ postinoculation was highest in bril-D, while $d 61-1$ and $d 2$ showed lower fungal biomass than WT plants (Fig. 1C), indicating that BR signaling negatively regulates rice resistance to sheath blight disease.

Because RAVL1 is a key transcription factor that directly activates the expression of BRII, a BR receptor gene, and BR synthetic genes (Je et al. 2010), ravll mutants are insensitive to BR. Therefore, the two ravll mutants (ravll-1 and ravll-2) and RAVL1 overexpression plants (RAVL1 OX and RAVL1-GFP OX) (Je et al. 2010) were further utilized to examine response to sheath blight disease. The ravll mutants showed responses to $R$. solani AG-1 similar to that of WT, whereas RAVL1 OX lines were more susceptible than WT (Fig. 2A). The percentage of leaf area covered with lesions was $40 \%$ in WTand ravll mutants and $52 \%$ in RAVL1 OX plants (Fig. 2B). In addition, $72 \mathrm{~h}$ postinoculation RAVL1 OX plants showed higher fungal biomass than WT and ravll mutants (Fig. 2C). The qRT-PCR performed to quantify the expression level of BRII (Os01g0718300) and D2 (Os01g0197100) in the leaves of 1-monthold WT, ravll-1, and ravll-2 plants at $0,24,48$, and $72 \mathrm{~h}$ after $R$. solani AG-1 inoculation indicated that BRII and D2 expressions were not altered by $R$. solani AG-1 infection, and were lower in ravll mutants than in WT (Supplementary Fig. S2).

Mutation of $R A V L 1$ suppresses resistance to sheath blight disease in the $\mathbf{d 6 1 - 1}$ mutant. Because RAVL1 directly activates the expression of BRII and BR biosynthetic genes, including $D 2$ (Je et al. 2010), $d 61-1$ and $d 2$ mutants showed resistance to sheath blight disease. However, ravll mutants showed a WT-like response to $R$. solani AG-1. Thus, we further examined the function of RAVL1 and BRIl by crossing ravll (susceptible) and a $d 61-1$ (less susceptible than WT) mutants, and inoculating their F8 offspring with $R$. solani AG-1. The ravl1/d61-1 plants were susceptible to $R$. solani AG-1, similar to that shown by ravll (Fig. 3), indicating that RAVL1 mutation suppressed $d 61-1$ resistance to $R$. solani AG-1.

Expression of ethylene-related genes differed between ravl1 and WT plants. Although RAVL1 is known as an activator of BRI1, ravll suppresses the phenotype of $d 61-1$, suggesting that
A

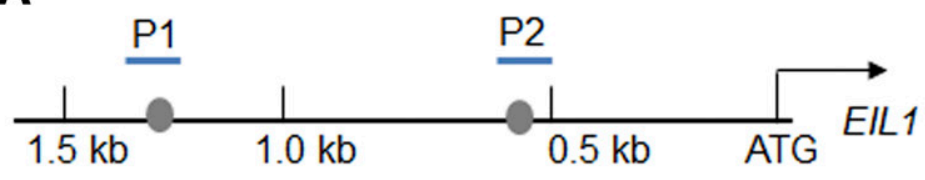

Putative E-box elements

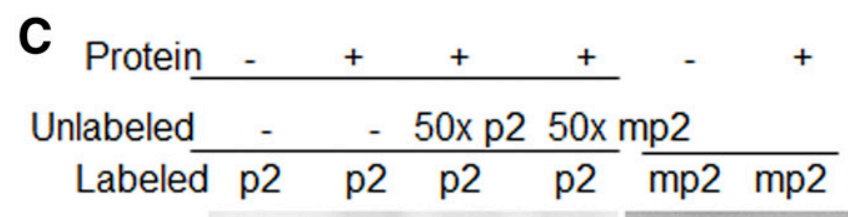

B
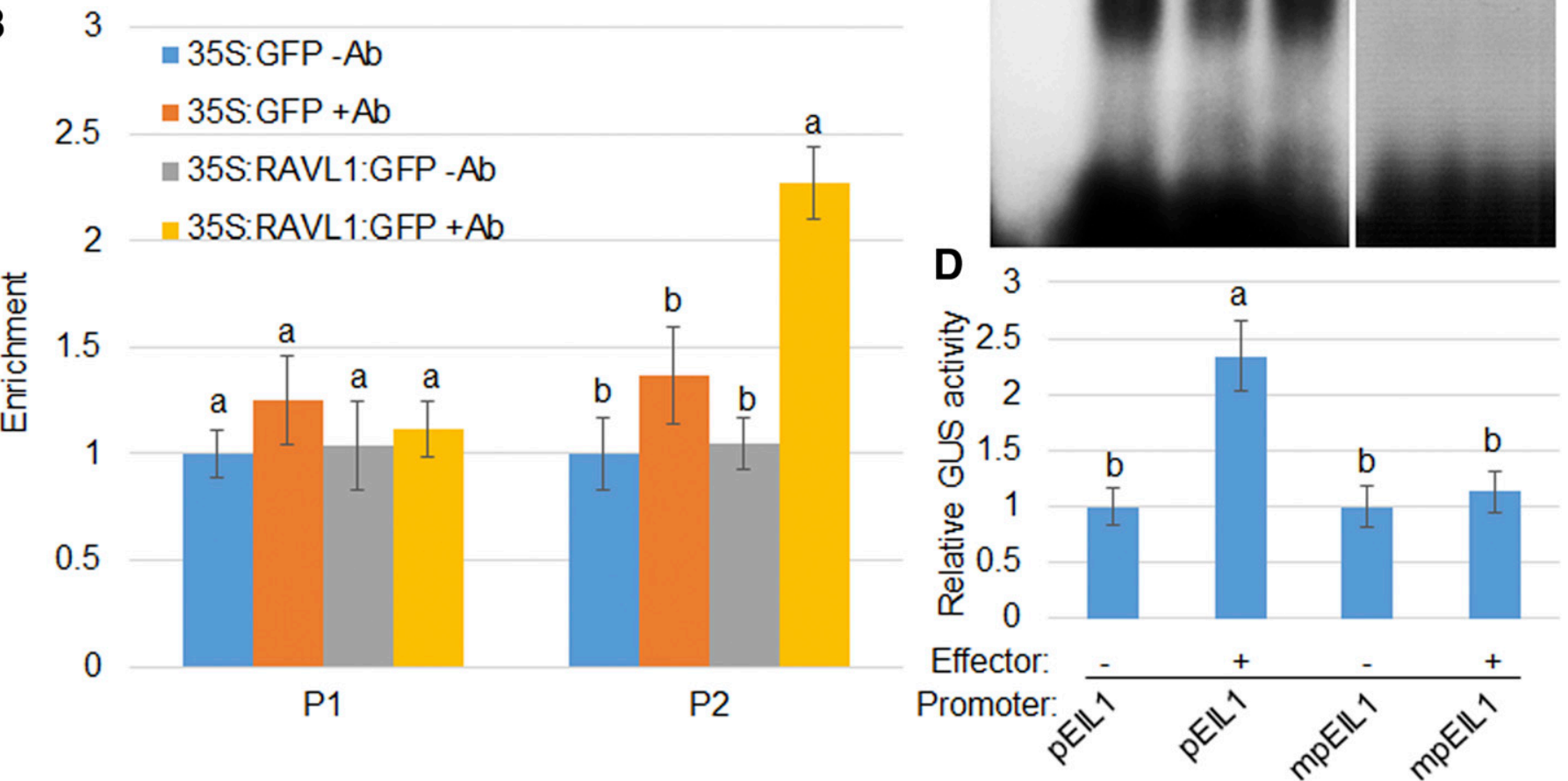

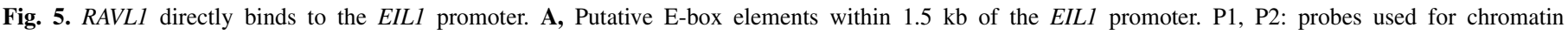

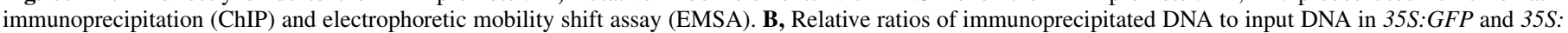

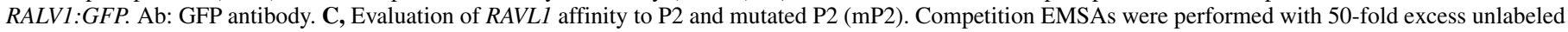

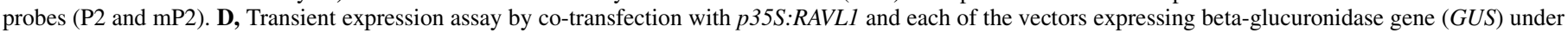

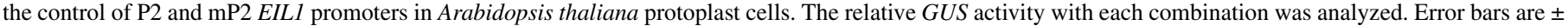
standard error of the mean $(n=6)$. GUS activity when expressing only $p E I L 1$ was defined as 1 . Different letters indicate significant differences at $P<0.05$. 
RAVL1 may also regulate other genes involved in sheath blight disease resistance. The transcriptome of 1-month-old WT and ravl1-1 mutant leaves was obtained to identify $R A V L 1$-regulated genes. Genes in ravll-1 mutants showing significantly altered expression (transcript levels presenting more than twofold changes in relation to levels in WT) were either upregulated (123 genes) or downregulated (86 genes) compared with WT ( $\log _{2} \geq 1$; Fig. 4A). These DEGs were involved in diverse biological processes, including transcription, carbohydrate metabolic process, transport, defense response, and hormone-mediated signaling (Supplementary Fig. S3). Six DEGs were ethylene-related genes whereas no BS-related gene was identified (Supplementary Table S2). A previous study showed that ethylene contributes to rice resistance against $R$. solani (Helliwell et al. 2013). Therefore, RAVL1 might positively regulate rice resistance against sheath blight disease through the ethylene signaling pathway. Based on this hypothesis, a qRT-PCR was performed to verify the expression levels of EIL1 (Os03g20790), ACO2 (Os09g27750), and three putative ethylene resistance genes, $O s 07 g 06300$ (ethylene-insensitive protein 2), Os07g06190 (ethylene-insensitive protein 2), and Os09g20350 (ethylene-responsive transcription factor) in WT, ravll-1 and ravll2, and RAVL1 OX and RAVL1-GFP OX. The levels of EIL1, Os07g06190, and Os09g20350 were lower in ravll mutants and higher in RAVL1 OX plants than in WT. However, the levels of
A

A

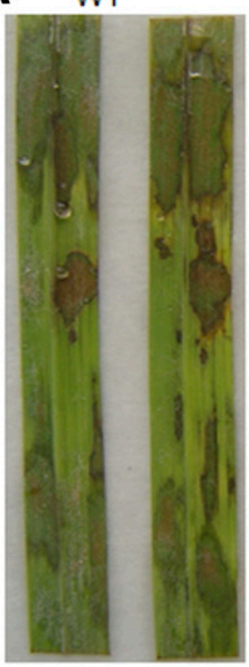

B

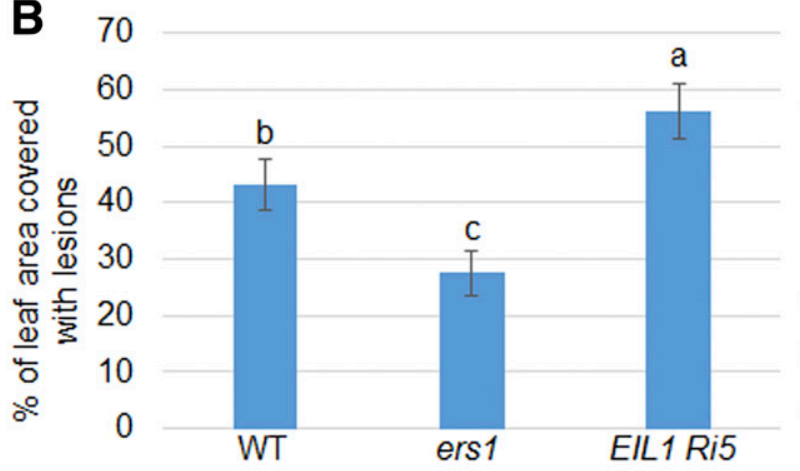

C 180

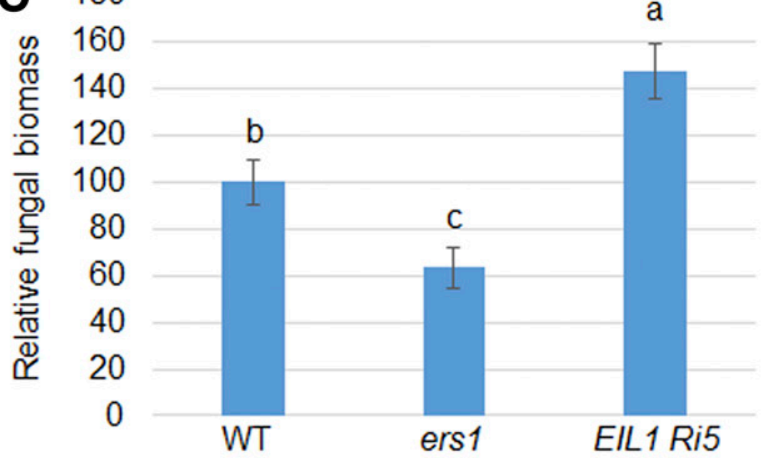

ers1
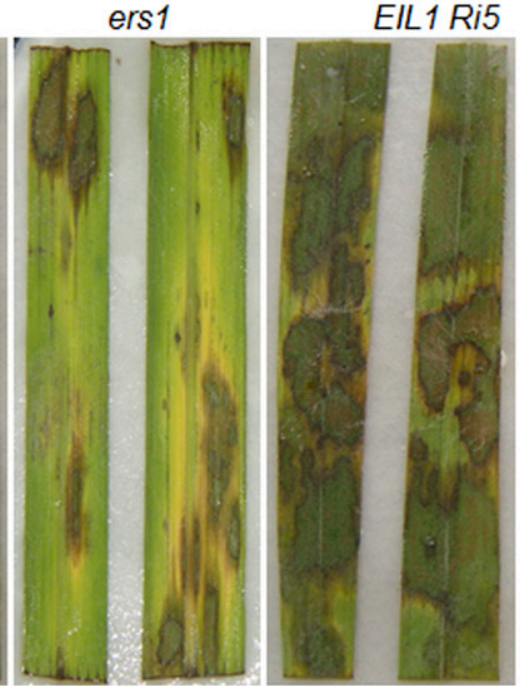

D

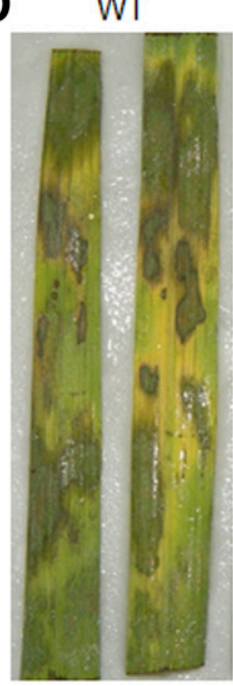

E 70

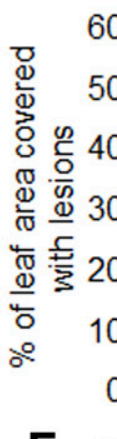

F 180

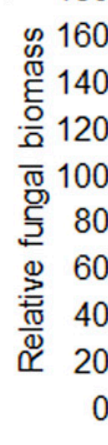

60

40

30

20

10

(0)

WT

WT $d 61-1$

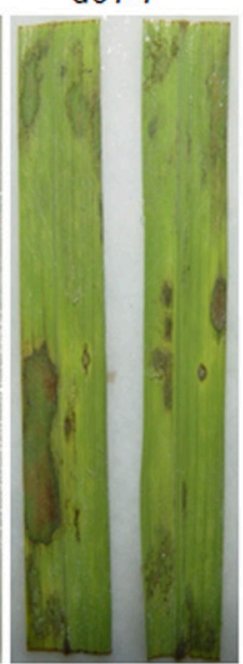

d61-1/EIL1 Ri5
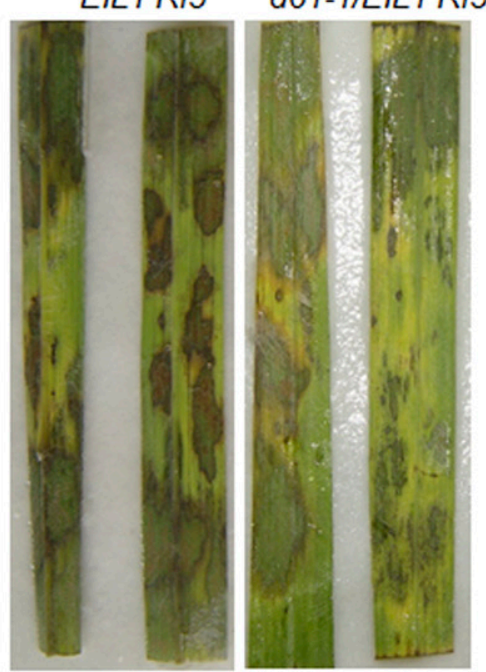

a

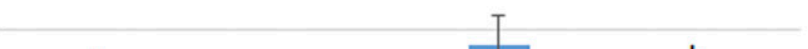

b $\quad$ b

WT $\quad d 61-1$

EIL1 Ri5

d61-1/EIL1 Ri5

a

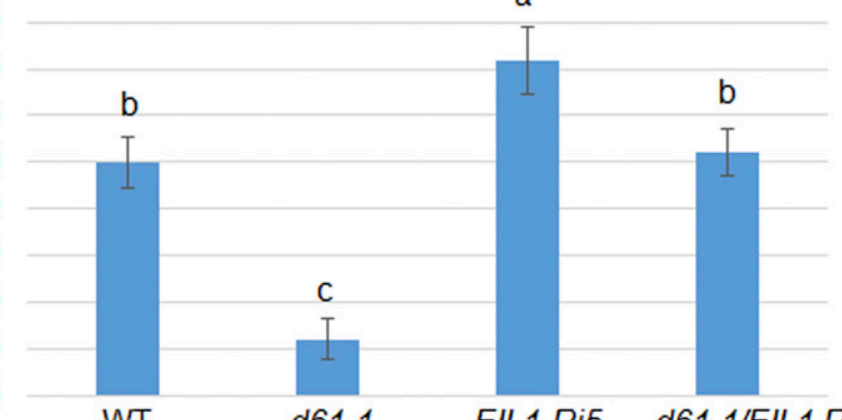

$d 61-1$

EIL1 Ri5

d61-1/EIL1 Ri5

Fig. 6. Evaluation of ers 1 and EIL1 Ri5, and d61-1/EIL1 Ri5 double mutants in response to sheath blight disease compared with wild-type (WT) rice (Oryza sativa L.). A, Response of ers1 and EIL1 Ri5 to Rhizoctonia solani anastomosis group (AG)-1 compared with WT. B, Percentage of leaf area covered with lesions in ers1 and EIL1 Ri5 compared with WT. Data represent means \pm standard error $(n>10)$. C, Relative fungal biomass obtained by quantitative PCR [2[CT $(O s U B I)$-CT $(R s P G D)]$ ] using genomic DNA. The data are means \pm standard error $(n=10)$. D, Response of d61-1/EIL1 Ri5 (F3) to R. solani AG-1 compared with WT and parental lines. E, Percentage of leaf area covered with lesions in d61-1/EIL Ri5 compared with WT and parental lines. Data represent means \pm standard error $(n>10)$. F, Relative fungal biomass obtained by quantitative PCR $\left[2^{[\mathrm{CT}(O s U B I)-\mathrm{CT}(R s P G D)]}\right]$ using genomic DNA. The data are means \pm standard error $(n=10)$. Different letters indicate significant differences at $P<0.05$. 
$A C O 2$ and $O s 07 g 06300$ were higher in ravll than in WT and RAVL1 OX (Fig. 4B), similar to that obtained by RNA sequencing. In addition, RAVLl (HM450152) was highly expressed in RAVLl OX compared with WT, but this transcript was not detected in ravll (Fig. 4B).

RAVL1 transcriptionally activates EILI directly via promoter binding. EIL1, Os07g06190, and Os09g20350 expressions were lower in ravll mutants and higher in RAVL1 OX's than in WT plants (Fig. 4B). The promoter sequences of EIL1, Os07g06190, and Os $09 g 20350$ were analyzed to identify the presence of possible binding sites for RAVL1. We identified two putative E-box elements located within $1.5 \mathrm{~kb}$ of the EILl promoter (Fig. 5A), whereas no putative E-box elements were observed within $1.5 \mathrm{~kb}$ of
Os07g06190 and Os09g20350 promoters (data not shown). The ChIP assay using 35S:GFP and 35S:RAVL1:GFP transgenic plant calli showed that precipitation of RAVL1 enriched the $\mathrm{P} 2$ region, but not the P1 region, of the EIL1 promoter (Fig. 5B). Additionally, EMSA results suggested that RAVL1 can bind to E-boxes in the P2 region, but not to E-box sequences mutated to TTTTTT (Fig. 5C). The competition EMSA performed to verify the binding affinity between RAVL1 and the P2 region revealed that this binding was greatly reduced in the presence of 50-fold excess unlabeled P2 but unaffected by unlabeled $\mathrm{mP2}$ (Fig. 5C). To verify if these ciselements were responsible for the transcriptional activation of the EIL1 promoter via RAVL1 in vivo, we performed a transactivation assay by transient expression in A. thaliana protoplasts. Results
A

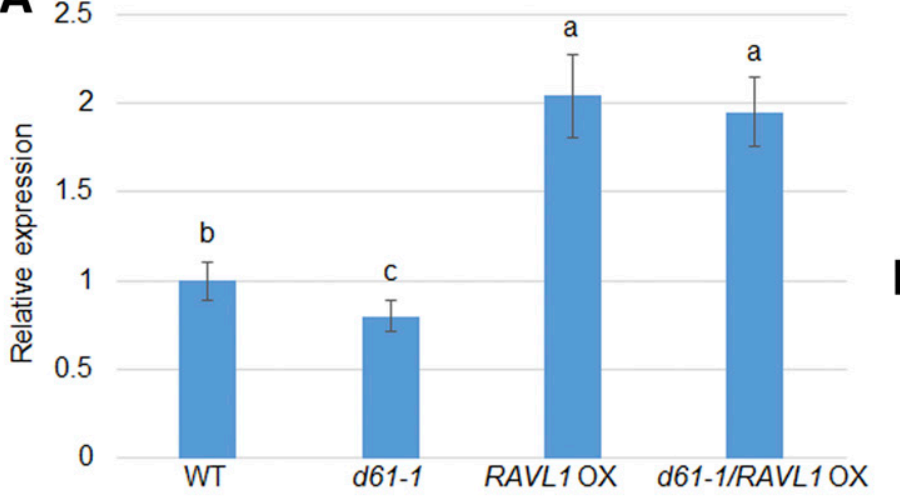

B

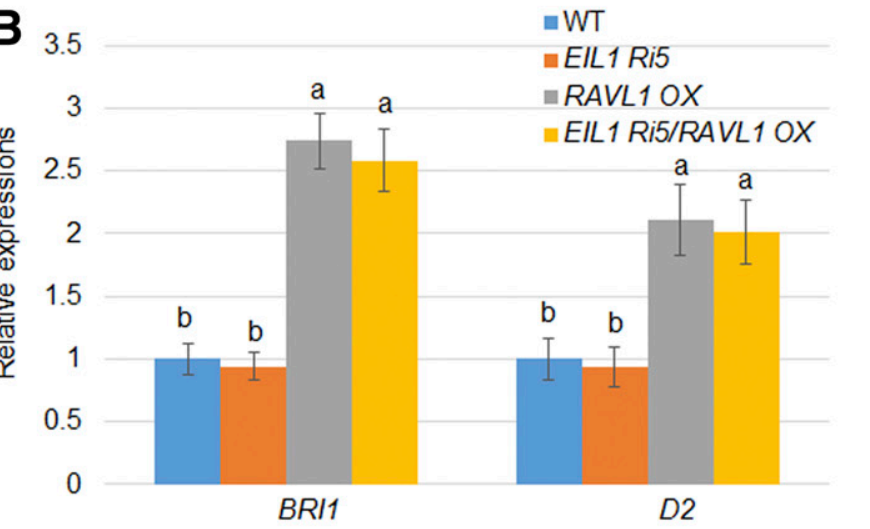

C

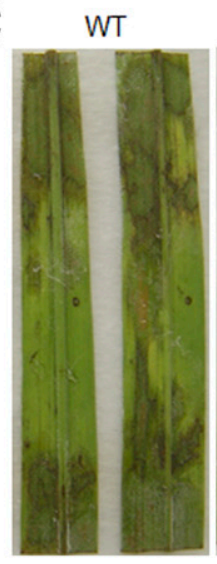

$d 61-1$

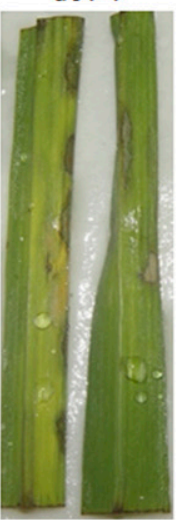

RAVL1OX d61-1/RAVL1OX
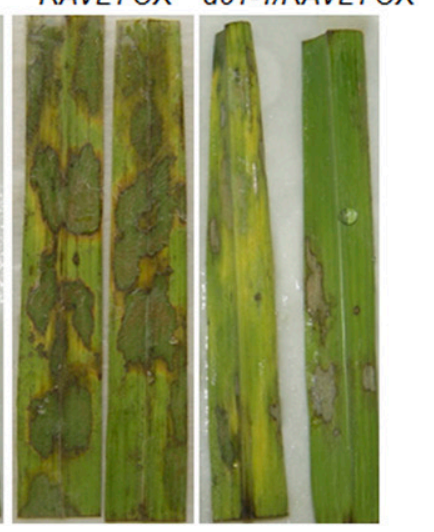

D

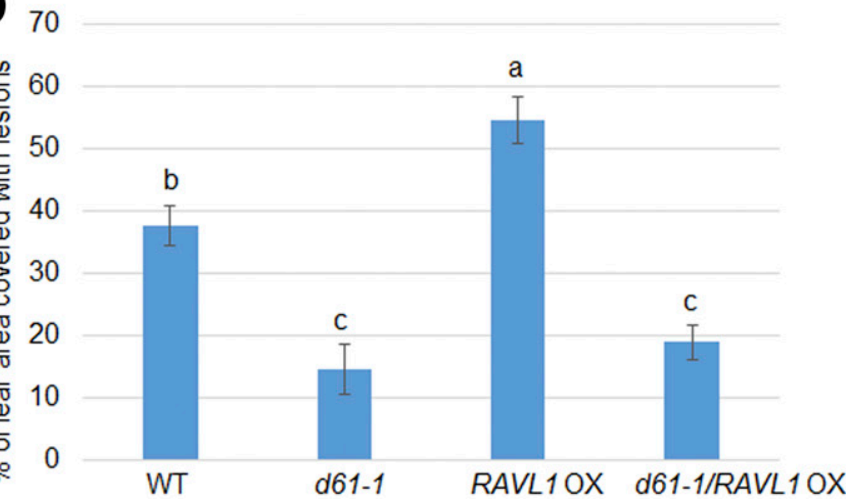

E 180

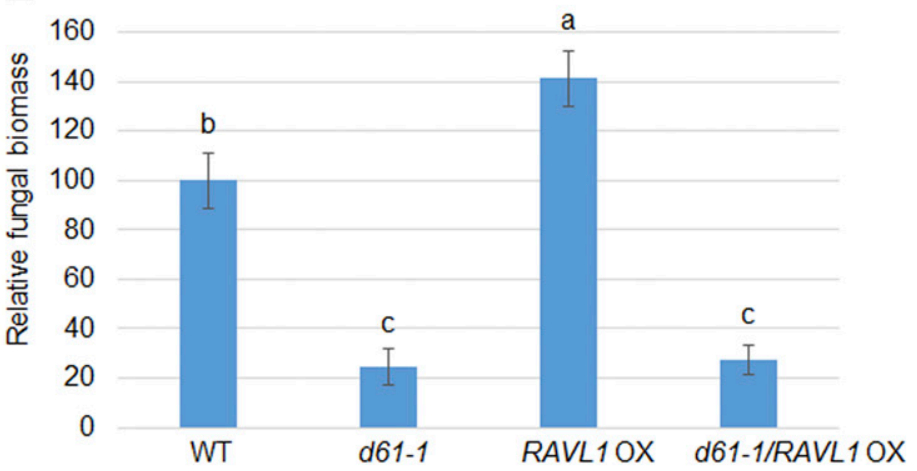

Fig. 7. Genetic and phenotypic evaluation of RAVL1 action on gene expressions and $d 61-1 / R A V L 1$ overexpressing (OX) double mutant in response to sheath blight disease compared with wild-type (WT) rice (Oryza sativa L.) and parental lines. A, Relative expression of EIL1 in d61-1/RAVL1 OX (F8) compared with WT and parental lines. B, Relative expression of BRI1 and D2 in EIL1 Ri5/RAVL1 OX (F3) compared with WT and parental lines. The mRNA levels of samples were normalized to that of Ubiquitin mRNA. Data represent means \pm standard error $(n=3)$. The expressions of EIL1, BRI1 and D2 in WT were defined as 1. C, Response of $d 61-1 /$ RAVL1 OX to Rhizoctonia solani anastomosis group (AG)-1 compared with WT and parental lines. D, Percentage of leaf area covered with lesions in $d 61-1 / R A V L 1$ OX compared with WT and parental lines. Data represent means \pm standard error $(n>10)$. E, Relative fungal biomass obtained by quantitative PCR [2 $\left.2^{[C T(O s U B I) \text {-CT }(R s P G D)]}\right]$ using genomic DNA. The data are means \pm standard error $(n=10)$. Different letters indicate significant differences at $P<0.05$. 
suggested that RAVL1 can transactivate $p E I L 1$, but not the E-box mutated promoter $m p E I L 1$ (Fig. 5D), indicating that RAVL1 might activate EILI expression via promoter binding.

Ethylene signaling positively regulates rice resistance to sheath blight disease. To test the function of EILI in response to $R$. solani AG-1, EIL1 RNAi transgenic plants were first analyzed by qRT-PCR for the detection of EIL1 expression; the results showed that EIL1 was suppressed by 50 to $80 \%$ (Supplementary Fig. S4A). Phenotypic evaluation of EIL1 RNAi in response to sheath blight disease revealed that transgenic plants were susceptible to $R$. solani AG-1. To confirm the role of ethylene signaling in response to sheath blight disease, we further analyzed the ethylene receptor ERS1 mutant ers1 and EIL1 RNAi 5 (EIL1 Ri5). The ers1 mutant showed a strong ethylene response phenotype to exogenous ethylene, indicating that ERS1 is a negative regulator of ethylene signaling (Ma et al. 2014). The results showed that ers 1 was more resistant to $R$. solani AG-1 than WT, whereas EIL1 RNAi 5 (EIL1 Ri5) was more susceptible than WT (Fig. 6A, B, and C), indicating that ethylene signaling positively regulates rice resistance to sheath blight disease.

EIL1 inhibition suppresses resistance to sheath blight disease in the d61-1 mutant. Because RAVL1 apparently activates the expression of EILI and BRII, two genes that have opposite functions in response to $R$. solani AG-1, we further examined the function of EILI and BRII by crossing EIL1 Ri5 (more susceptible than WT) and $d 61-1$ (more resistant than WT) mutants and inoculating their F3 offspring with $R$. solani AG-1. The results showed that $d 61-1 / E I L 1 R i 5$ was susceptible to $R$. solani AG-1, similar to WT (Fig. 6D, E, and F), indicating that EIL1 suppression reduced $d 61-1$ resistance to sheath blight disease (Fig. $6 \mathrm{D}$, E, and F).

RALV1 coordinates $B R$ and ethylene signaling. Because RAVL1 directly activates the expression of BR and ethylene-related genes, we examined if the activation of EILI by $R A V L 1$ required BR signaling and if the RAVL1 activation of BRII and D2 required ethylene signaling. To this end, we monitored the levels of EIL1 in RAVL1 OX/d61-1 and those of BRII and D2 in RAVL1 OX/ EIL1 Ri5. The results showed that levels of EIL1 were slightly lower in $d 61-1$, but markedly higher in RAVL1 OX and $d 61-1 / R A V L 1$ OX (F8), than in WT (Fig. 7A). Additionally, the levels of BRI1 and D2 were significantly higher in RAVL1 OX and EIL1 Ri5/RAVL1 OX (F3) than in WT and EIL1 Ri5 (Fig. 7B). Further, BRI1 mutation enhanced resistance to $R$. solani AG-1 in a RAVL1 OX background, with $d 61-1 / R A V L 1$ OX showing a phenotype similar to that of $d 61-1$ (Fig. 7C, D, and E), indicating that the susceptibility of RAVL1 OX to $R$. solani AG-1 depends on BR signaling.

\section{DISCUSSION}

Sheath blight disease caused by $R$. solani is a major rice disease, which severely reduces grain yield; however, the host resistance mechanisms remain unknown. Previous studies have shown that BR signaling is closely associated with host-pathogen interaction (Ali et al. 2013; Goddard et al. 2014). The present study showed that the BRIl mutant $d 61-1$ and the key BR biosynthesis gene mutant $d 2$ are highly resistant to $R$. solani AG-1, whereas the BRII overexpression line bril-D is more susceptible than WT plants, indicating that BR signaling supports rice susceptibility against sheath blight disease. Furthermore, analyses of the key BR signaling transcription factor RAVL1 in response to sheath blight disease showed that ravll mutants exhibited similar symptoms to WT plants, whereas RAVLI OX plants showed enhanced susceptibility, suggesting that ravll mutants might regulate other genes besides BR signaling genes, or that a protein functionally redundant to RAVL1 might modulate the development of sheath blight disease together with RAVL1.

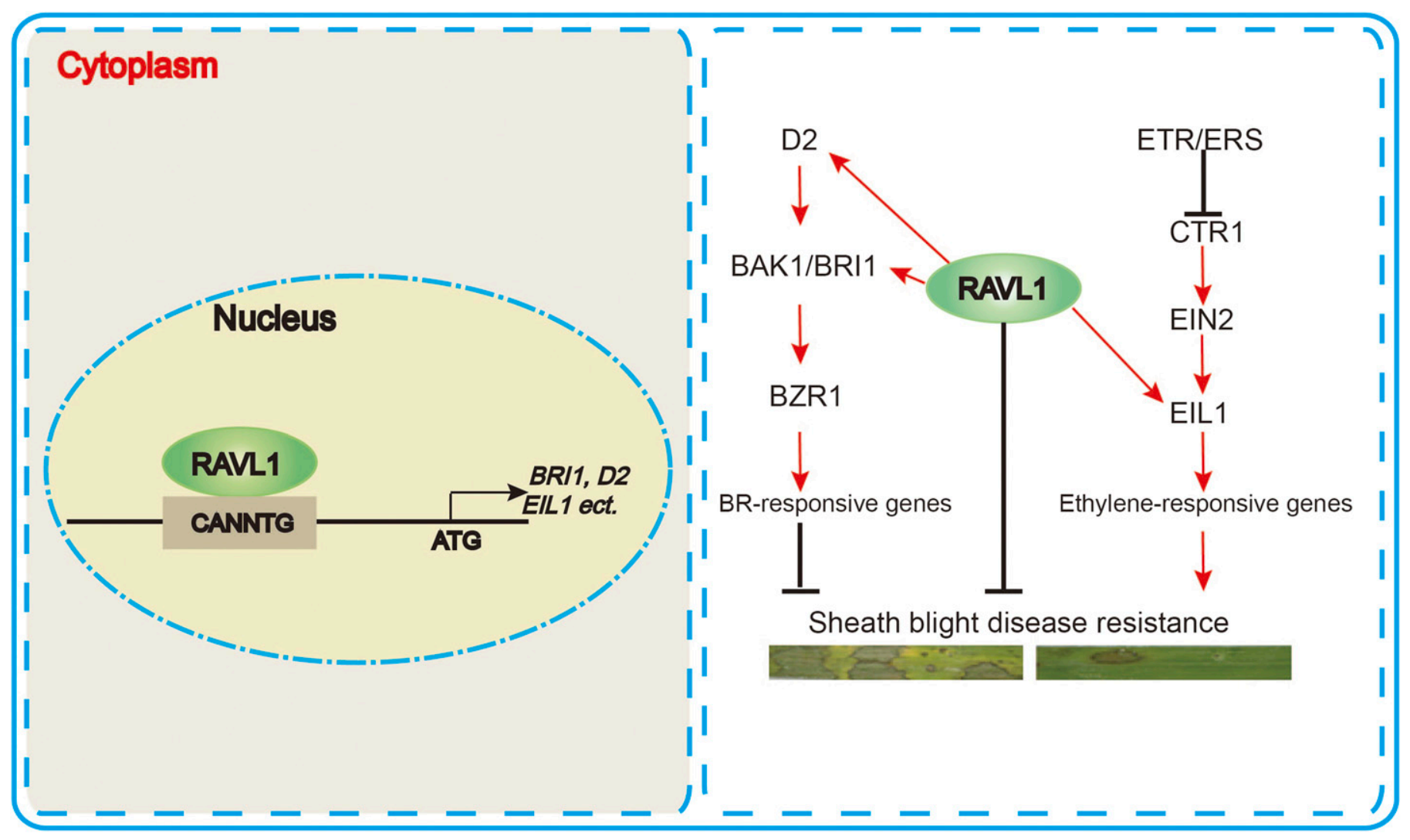

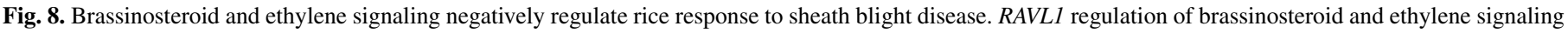
in response to sheath blight disease. RAVL1 directly activates brassinosteroid- (BRII and D2) and ethylene- (EIL1) related genes. 
In the present study, transcriptome analysis of WT and ravll plants revealed that the expression of few ethylene-related genes, including EIL1 and ACO2, was altered in ravll. However, no BRrelated gene showed more than twofold changes in expression in ravll. Further verification via qRT-PCR demonstrated that EIL1 was positively regulated by RAVL1. The promoter sequence analysis identified two E-box elements located within $1.5 \mathrm{~kb}$ of the EIL1 promoter. Additionally, the ChIP assay suggested that RAVL1 can bind to P2 elements, which are located 688-bp downstream of the start codon, but not to the $\mathrm{P} 1$ region. The in vitro EMSA performed to verify if RAVL1 directly bound to P2 indicated that the RAVL1 recombinant protein bound to $\mathrm{P} 2$, but not to E-box element sequences mutated to TTTTTT. Further competition EMSA experiments suggested that RAVL1 specifically bound the P2 region. The transient promoter transactivation assay supported that RALV1 can directly activate expression of EILI via promoter binding. The levels of EIL1, $O s 07 g 06190$, and $O s 09 g 20350$ were lower in ravll mutants and higher in RAVL1 OX than in WT, while the levels of ACO2 and $O s 07 \mathrm{gO6300}$ were higher in ravll than in WT and RAVL1 OX. Although EIL1 is known as a positive regulator, the role of Os07g06190, Os09g20350, and $0 s 07 g 06300$ in ethylene signaling is unclear. RAVL1 activates EIL1, and ravll mutants are less sensitive while RAVL1 OX is more sensitive to ACC than WT, suggesting that RAVL1 might positively regulate ethylene signaling.

Previous studies revealed the interaction between BR and ethylene signaling (Gendron et al. 2008), and showed that BR treatment elevates ethylene production by increasing the stability of acetyl-coenzyme A synthetase (ACS), suggesting a potential role for BRs in the regulation of ethylene biogenesis (Hansen et al. 2009). However, direct associations between BR and ethylene signaling have not been identified. Our data suggest that BR and ethylene signaling are linked by RAVL1. A previous study reported that the overexpression of $O s A C S 2$, an ethylene biosynthetic gene, enhances rice resistance to rice blast and sheath blight disease (Helliwell et al. 2013). Because EIL1 is a major regulator of ethylene signaling, the response of EIL1 RNA $i$ lines against $R$. solani AG-1 was tested. The expression levels of EILI in RNAi plants were significantly suppressed, and thus, the plants were more susceptible to $R$. solani AG-1 than WT. Additionally, the ethylenehypersensitive ers 1 mutant was examined, and data showed that it was more resistant than WT. Therefore, ethylene signaling positively regulates rice resistance to sheath blight disease.

We found that RAVL1 transcriptionally regulates both BR and ethylene signaling genes and that BR and ethylene signaling oppositely regulated plant resistance to sheath blight disease. Whereas ravll exhibited infection symptoms similar to those of WT, $d 61-1$ was resistant to $R$. solani AG-1. Thus, a potential redundant protein of RAVL1 might exist or other RAVL1 downstream gene functionally opposite to BRII might respond to $R$. solani AG- 1 . Therefore, we examined whether these effects were caused by downregulation of both BRII and EILI. To this end, we genetically combined BRII and EIL1 by crossing d61-1 and EIL1 RNAi 5 (EIL1 Ri5) mutants and found that $d 61-1 / E I L 1$ Ri5 had a similar phenotype to that of WT. Thus, combined BR and ethylene signaling modulate rice defense against sheath blight disease. To examine if the RAVLI activation of EIL1 depends on BR signaling, the expression levels of EIL1 were examined in RAVL1 OX and $d 61-1 / R A V L 1$ OX. Results showed that EIL1 expression was significantly higher in both RAVL1 OX and $d 61$ $1 / R A V L 1$ OX than in WT, indicating that RAVL1 transcriptionally activates EIL1 independently of BR signaling. Similarly, the expression levels of BRII and D2 examined in RAVL1 OX and EIL1 Ri5/ $R A V L 1$ OX indicated that BRII and $D 2$ were highly expressed in RAVL1 OX and EIL1 Ri5/RAVL1 OX. Thus, RAVL1 in BR and ethylene signaling are involved rice defense against sheath blight disease (Fig. 8).

Both ravll mutants were WT-like and RAVL1 OX was more susceptible than WT to $R$. solani AG-1, suggesting that overexpression of $R A V L 1$ might transcriptionally activate genes that do not change their action in ravll. Further, the RAVLI OX phenotype disappeared in $661-1 / R A V L 1$ OX, suggesting that BRII is epistatic to $R A V L 1$ OX in response to $R$. solani AG-1. Furthermore, BRII and $D 2$ expression was not affected upon $R$. solani infection, suggesting that RAVL1 might contribute to BR and ET signaling pathway differentially under different conditions, such as plant development and resistance. Overall, the present study provides information on the role of $\mathrm{BR}$ and ethylene signaling in response to sheath blight disease, and identifies RAVL1 as the linkage between $\mathrm{BR}$ and ethylene signaling in rice.

\section{LITERATURE CITED}

Agrawal, G. K., Rawkwal, R., and Jwa, N. S. 2001. Differential induction of three pathogenesis-related genes, $P R 10, P R 1 b$, and $P R 5$ by the ethylene generator ethephon under light and dark in rice (Oryza sativa L.) seedlings. J. Plant Physiol. 158:133-137.

Ali, S. S., Kumar, G. B., Khan, M., and Doohan, F. M. 2013. Brassinosteroid enhances resistance to Fusarium diseases of barley. Phytopathology 103: 1260-1267.

Anderson, J. P., Lichtenzveig, J., Gleason, C., Oliver, R. P., and Singh, K. B. 2010. The B-3 ethylene response factor MtERF1-1 mediates resistance to a subset of root pathogens in Medicago truncatula without adversely affecting symbiosis with rhizobia. Plant Physiol. 154:861-873.

Chang, C., Kwok, S. F., Bleecker, A. B., and Meyerowitz, E. M. 1993. Arabidopsis ethylene-response gene ETR1: Similarity of product to twocomponent regulators. Science 262:539-544.

Dunnick, J., Brix, A., Cunny, H., Vallant, M., and Shockley, K. 2012. Characterization of polybrominated diphenyl ether toxicity in Wistar Han rats and use of liver microarray data for predicting disease susceptibilities. Toxicol. Pathol. 40:93-106.

Dupuy, D., Bertin, N., Hidalgo, C. A., Venkatesan, K., Tu, D., Lee, D., Rosenberg, J., Svrzikapa, N., Blanc, A., Carnec, A., Carvunis, A. R., Pulak, R., Shingles, J., Reece-Hoyes, J., Hunt-Newbury, R., Viveiros, R., Mohler, W. A., Tasan, M., Roth, F. P., Le Peuch, C., Hope, I. A., Johnsen, R., Moerman, D. G., Barabási, A. L., Baillie, D., and Vidal, M. 2007. Genomescale analysis of in vivo spatiotemporal promoter activity in Caenorhabditis elegans. Nat. Biotechnol. 25:663-668.

Eizenga, G. C., Lee, F. N., and Rutger, J. N. 2002. Screening Oryza species plants for rice sheath blight resistance. Plant Dis. 86:808-812.

Gendron, J. M., Haque, A., Gendron, N., Chang, T., Asami, T., and Wang, Z. Y. 2008. Chemical genetic dissection of brassinosteroid-ethylene interaction. Mol. Plant 1:368-379.

Goddard, R., Peraldi, A., Ridout, C., and Nicholson, P. 2014. Enhanced disease resistance caused by BRII mutation is conserved between Brachypodium distachyon and barley (Hordeum vulgare). Mol. Plant-Microbe Interact. 27: 1095-1106.

Hansen, M., Chae, H. S., and Kieber, J. J. 2009. Regulation of ACS protein stability by cytokinin and brassinosteroid. Plant J. 57:606-614.

Helliwell, E. E., Wang, Q., and Yang, Y. 2013. Transgenic rice with inducible ethylene production exhibits broad-spectrum disease resistance to the fungal pathogens Magnaporthe oryzae and Rhizoctonia solani. Plant Biotechnol. J. $11: 33-42$.

Hong, Z., Ueguchi-Tanaka, M., Umemura, K., Uozu, S., Fujioka, S., Takatsuto, S., Ashikari, M., Kitano, H., and Matsuoka, M. 2003. A rice brassinosteroid-deficient mutant, ebisu dwarf (d2), is caused by a loss of function of a new member of cytochrome p450. Plant Cell 15:2900-2910.

Hua, J., Chang, C., Sun, Q., and Meyerowitz, E. M. 1995. Ethylene insensitivity conferred by Arabidopsis ERS gene. Science 269:1712-1714.

Je, B. I., Piao, H. L., Park, S. J., Park, S. H., Kim, C. M., Xuan, Y. H., Park, S. H., Huang, J., Choi, Y. D., An, G., Wong, H. L., Fujioka, S., Kim, M. C., Shimamoto, K., and Han, C. D. 2010. RAV-Like1 maintains brassinosteroid homeostasis via the coordinated activation of BRII and biosynthetic genes in rice. Plant Cell 22:1777-1791.

Jeong, D. H., An, S., Kang, H. G., Moon, S., Han, J. J., Park, S., Lee, H. S., An, K., and An, G. 2002. T-DNA insertional mutagenesis for activation tagging in rice. Plant Physiol. 130:1636-1644.

Ju, C., Yoon, G. M., Shemansky, J. M., Lin, D. Y., Ying, Z. I., Chang, J., Garrett, W. M., Kessenbrock, M., Groth, G., Tucker, M. L., Cooper, B., Kieber, J. J., and Chang, C. 2012. CTR1 phosphorylates the central regulator EIN2 to control ethylene hormone signaling from the ER membrane to the nucleus in Arabidopsis. Proc. Natl. Acad. Sci. USA 109:19486-19491.

Kieber, J. J., Rothenberg, M., Roman, G., Feldmann, K. A., and Ecker, J. R. 1993. CTR1, a negative regulator of the ethylene response pathway in Arabidopsis, encodes a member of the raf family of protein kinases. Cell 72:427-441.

Kim, T. W., and Wang, Z. Y. 2010. Brassinosteroid signal transduction from receptor kinases to transcription factors. Annu. Rev. Plant Biol. 61:681-704. 
Li, J., and Chory, J. 1997. A putative leucine-rich repeat receptor kinase involved in brassinosteroid signal transduction. Cell 90:929-938.

Li, J., Wen, J., Lease, K. A., Doke, J. T., Tax, F. E., and Walker, J. C. 2002. BAK1, an Arabidopsis LRR receptor-like protein kinase, interacts with BRI1 and modulates brassinosteroid signaling. Cell 110:213-222.

Ma, B., Yin, C. C., He, S. J., Lu, X., Zhang, W. K., Lu, T. G., Chen, S. Y., and Zhang, J. S. 2014. Ethylene-induced inhibition of root growth requires abscisic acid function in rice (Oryza sativa L.) seedlings. PLoS Genet. 10: e1004701.

Mao, B., Liu, X., Hu, D., and Li, D. 2014. Co-expression of $\mathrm{RCH} 10$ and AGLU1 confers rice resistance to fungal sheath blight Rhizoctonia solani and blast Magnaporthe oryzae and reveals impact on seed germination. World J. Microbiol. Biotechnol. 30:1229-1238.

Morgan, P. W., and Drew, M. C. 1997. Ethylene and plant responses to stress. Physiol. Plant. 100:620-630.

Nam, K. H., and Li, J. 2002. BRI1/BAK1, a receptor kinase pair mediating brassinosteroid signaling. Cell 110:203-212.

Prasad, B., and Eizenga, G. C. 2008. Rice sheath blight disease resistance identified in Oryza spp. Plant Dis. 92:1503-1509.

Qiao, H., Shen, Z., Huang, S. S., Schmitz, R. J., Urich, M. A., Briggs, S. P., and Ecker, J. R. 2012. Processing and subcellular trafficking of ER-tethered EIN2 control response to ethylene gas. Science 338:390-393.

Sakai, H., Hua, J., Chen, Q. G., Chang, C., Medrano, L. J., Bleecker, A. B., and Meyerowitz, E. M. 1998. ETR2 is an ETR1-like gene involved in ethylene signaling in Arabidopsis. Proc. Natl. Acad. Sci. USA 95:5812-5817.

Savary, S., Castilla, N. P., Elazegui, F. A., McLaren, C. G., Ynalvez, M. A., and Teng, P. S. 1995. Direct and indirect effects of nitrogen supply and disease source structure on rice sheath blight spread. Phytopathology 85: 959-965.

Savary, S., and Mew, T. W. 1996. Analyzing crop losses due to Rhizoctonia solani: Rice sheath blight, a case study. Pages 237-245 in: Rhizoctonia Species: Taxonomy, Molecular Biology, Ecology, Pathology and Disease Control. Springer, Dordrecht, the Netherlands.

Savary, S., Willocquet, L., Elazegui, F. A., Castilla, N. P., and Teng, P. S. 2000. Rice pest constraints in tropical Asia: Quantification of yield losses due to rice pests in a range of production situations. Plant Dis. 84:357-369.

Shah, J. M., Raghupathy, V., and Veluthambi, K. 2009. Enhanced sheath blight resistance in transgenic rice expressing an endochitinase gene from Trichoderma virens. Biotechnol. Lett. 31:239-244.

van Loon, L. C., Geraats, B. P., and Linthorst, H. J. 2006. Ethylene as a modulator of disease resistance in plants. Trends Plant Sci. 11:184-191.

Wang, R., Lu, L., Pan, X., Hu, Z., Ling, F., Yan, Y., Liu, Y., and Lin, Y. 2015. Functional analysis of $O S P G I P 1$ in rice sheath blight resistance. Plant Mol. Biol. 87:181-191.
Wang, Z. Y., Nakano, T., Gendron, J., He, J., Chen, M., Vafeados, D., Yang, Y., Fujioka, S., Yoshida, S., Asami, T., and Chory, J. 2002. Nuclear-localized BZR1 mediates brassinosteroid-induced growth and feedback suppression of brassinosteroid biosynthesis. Dev. Cell 2:505-513.

Wen, X., Zhang, C., Ji, Y., Zhao, Q., He, W., An, F., Jiang, L., and Guo, H. 2012. Activation of ethylene signaling is mediated by nuclear translocation of the cleaved EIN2 carboxyl terminus. Cell Res. 22:1613-1616.

Xuan, Y. H., Duan, F. Y., Je, B. I., Kim, C. M., Li, T. Y., Liu, J. M., Park, S. J., Cho, J. H., Kim, T. H., von Wiren, N., and Han, C. D. 2017. Related to $\mathrm{ABI}$ 3/VP1-Like $1(R A V L 1)$ regulates brassinosteroid-mediated activation of $A M T 1 ; 2$ in rice (Oryza sativa). J. Exp. Bot. 68:727-737.

Xuan, Y. H., Priatama, R. A., Huang, J., Je, B. I., Liu, J. M., Park, S. J., Piao, H. L., Son, D. Y., Lee, J. J., Park, S. H., Jung, K. H., Kim, T. H., and Han, C. D. 2013. Indeterminate domain 10 regulates ammonium-mediated gene expression in rice roots. New Phytol. 197:791-804.

Yamaguchi, M., Ohtani, M., Mitsuda, N., Kubo, M., Ohme-Takagi, M., Fukuda, H., and Demura, T. 2010. VND-INTERACTING 2 , a NAC domain transcription factor, negatively regulates xylem vessel formation in Arabidopsis. Plant Cell 22:1249-1263.

Yamamuro, C., Ihara, Y., Xiong, W., Noguchi, T., Fujioka, S., Takatsuto, S., Ashikari, M., Kitano, H., and Matsuoka, M. 2000. Loss of function of a rice brassinosteroid insensitive 1 homolog prevents internode elongation and bending of the lamina joint. Plant Cell 12:1591-1606.

Yang, C., Lu, X., Ma, B., Chen, S. Y., and Zhang, J. S. 2015a. Ethylene signaling in rice and Arabidopsis: Conserved and diverged aspects. Mol. Plant 8:495-505.

Yang, C., Ma, B., He, S. J., Xiong, Q., Duan, K. X., Yin, C. C., Chen, H., Lu, X., Chen, S. Y., and Zhang, J. S. 2015b. MAOHUZI6/ETHYLENE $I_{N S E N S I T I V E}-L_{3} I K E_{1}$ and ETHYLENE INSENSITIVE ${ }_{3}-L_{I K E_{2}}$ regulate ethylene response of roots and coleoptiles and negatively affect salt tolerance in rice. Plant Physiol. 169:148-165.

Yang, C. J., Zhang, C., Lu, Y. N., Jin, J. Q., and Wang, X. L. 2011. The mechanisms of brassinosteroid action: From signal transduction to plant development. Mol. Plant. 4:588-600.

Ye, J., Fang, L., Zheng, H., Zhang, Y., Chen, J., Zhang, Z., Wang, J., Li, S., Li, R., Bolund, L., and Wang, J. 2006. WEGO: A web tool for plotting GO annotations. Nucleic Acids Res. 34:W293-W297.

Yin, Y., Wang, Z. Y., Mora-Garcia, S., Li, J., Yoshida, S., Asami, T., and Chory, J. 2002. BES1 accumulates in the nucleus in response to brassinosteroids to regulate gene expression and promote stem elongation. Cell 109:181-191.

Yoo, S. D., Cho, Y. H., and Sheen, J. 2007. Arabidopsis mesophyll protoplasts: A versatile cell system for transient gene expression analysis. Nat. Protoc. 2:1565-1572. 